\title{
Strip inverse-configuration photopyroelectric technique to measure the thermal conductivity of bulk samples
}

\author{
Srirang Manohar and S. Asokan ${ }^{\mathrm{a})}$ \\ Department of Instrumentation, Indian Institute of Science, Bangalore 560012, India
}

\begin{abstract}
The strip inverse-configuration photopyroelectric technique to measure the thermal conductivity of bulk samples of metals, semiconductors, and insulators is presented. It utilizes a narrow $(500 \mu \mathrm{m}$ wide) strip of a $9 \mu \mathrm{m}$ thick pyroelectric sensor in an inverse configuration. A mathematical model is outlined that yields an expression for the output voltage of the pyroelectric sensor following heat dissipation into the sample, in a frequency range where the proposed measurement is possible. Experimentally, the sensor was operated in an "isothermal-voltage" mode to identify the frequency regime within which the measurement of the thermal conductivity of representative samples was possible after suitable calibration of the system.
\end{abstract}

Photopyroelectric (PPE) experiments rely on pyroelectric sensor based detection of heat generated in a sample due to nonradiative deexcitation processes of the absorbed optical energy. ${ }^{1,2}$ Spectroscopic investigations can be conducted by monitoring the wavelength dependence of such heating in the specimen. Additionally, the use of incident radiation to produce a localized heat source in the sample permits calorimetric studies as well.

In conventional frequency domain calorimetric PPE techniques, the periodic nature of the excitation lends itself naturally to a measure of the thermal diffusivity $(\alpha)$ as a primary quantity, usually from the signal phase. ${ }^{3}$ The thermal effusivity $(e)$, is obtained simultaneously from the signal amplitude ${ }^{3}$ the thermal conductivity $(\kappa)$ is then calculated by using the relationship between $e$ and $\alpha$.

In this letter, we present a frequency domain technique that we refer to as the strip inverse-configuration photopyroelectric (STRIPPE) method, which can be used under some conditions, to obtain a direct measure of the thermal conductivity of bulk samples. The technique is based on heat diffusion into the specimen from a long narrow strip of a thin pyroelectric sensor operated in the inverse configuration, ${ }^{4}$ where it also serves as the heat source when illuminated with intensity modulated light. In previous variants of the PPE technique, a two-dimensional scheme was not sought and the geometry of the sensor and sample so configured that a onedimensional analysis of heat flow served the purpose well. In the present technique, the geometry is deliberately designed to obtain radial heat flow into the specimen under study by making the pyroelectric sensor/heater narrow. In such an arrangement, when the wavelength of the diffusive thermal wave in the sample is greater than the half-width of the strip, the temperature gradient established in the sample permits the measurement of $\kappa$ by monitoring the output of the pyroelectric.

A side view of the pyroelectric strip and the sample is shown in Fig. 1. It is assumed that the free surface of the

${ }^{a)}$ Electronic mail: sasokan@isu.iisc.ernet.in sample at $y=0$ and that of the pyroelectric at $y=-l_{p}$ is bounded by vacuum. The following are the other assumptions of the model: (a) The top electrode of the pyroelectric strip is highly absorbing but thin enough so that it can be replaced by a plane source of heat; (b) the strip is illuminated uniformly with light, the intensity of which is modulated sinusoidally at a frequency of $\omega \mathrm{rad} / \mathrm{s}$ and the width (2a) of the strip is greater than its thickness $\left(l_{p}\right)$ so that a onedimensional equation can be used to describe heat flow in this region; (c) the pyroelectric strip is in good thermal contact with the sample, and temperature and heat flux continuity are experienced at the interface; and (d) radial heat flow occurs in the sample (which is semi-infinite) and a twodimensional heat equation is used in this region.

The heat conduction equations are

$$
\begin{array}{r}
\frac{d^{2}}{d y^{2}} T_{p}(\omega, y)-\frac{j \omega}{\alpha_{p}} T_{p}(\omega, y)=0, \quad\left[-l_{p} \leqslant y \leqslant 0\right], \\
\frac{\partial^{2}}{\partial y^{2}} T_{s}(\omega, x, y)+\frac{\partial^{2}}{\partial x^{2}} T_{s}(\omega, x, y)=\frac{j \omega}{\alpha_{s}} T_{s}(\omega, x, y), \\
\quad[-\infty<x<\infty, 0 \leqslant y<\infty] ;
\end{array}
$$

the boundary conditions $(\mathrm{BCs})$ are
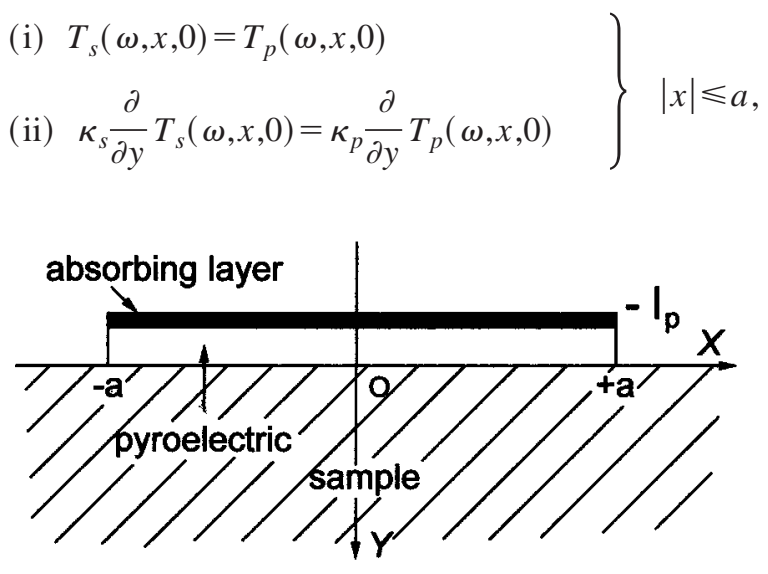

FIG. 1. Side view of the configuration. Light illuminates the top surface of the pyroelectric strip. 

(iii) $\left.\frac{\partial}{\partial y} T_{s}(\omega, x, 0)=0\right\}|x| \geqslant a$,
(iv) $\frac{\partial}{\partial y} T_{p}\left(\omega, x,-l_{p}\right)=Q_{a}$,
(v) $\left|T_{s}(\omega, x, y)\right| \rightarrow 0$ as $\sqrt{x^{2}+y^{2}} \rightarrow \infty$,

where $T_{i}$ is the temperature, $\alpha_{i}$ is the thermal diffusivity, and $\kappa_{i}$ is the thermal conductivity with $i=p$ or $s$, i.e., the pyroelectric or sample, as appropriate to the region. In addition to the above, since the model has symmetry about $x=0$ along the $Y$ axis, the requirement, $\partial / \partial x\left[T_{s}(\omega, 0, y)\right]=0$ can be introduced as boundary condition (vi). In the above, the term $Q_{a}$ refers to the heat source obtained due to absorption at the top electrode. It should be noted that (i) or (ii) along with (iii) describe different conditions that prevail over different sections of the same boundary. ${ }^{5}$

The BC (vi) immediately suggests the use of the Fourier cosine (FC) transform ${ }^{5}$ with respect to $x$ for the solution of Eq. (2). On inversion and having discarded one of the constants in the general solution, in obedience with BC (v), and incorporating $\mathrm{BCs}$ (ii) and (iii) above requires the remaining constant $A(\omega, \xi)$ to be the solution of the dual integral equations $^{5}$

$$
\begin{array}{r}
-\sqrt{\frac{2}{\pi}} \kappa_{s} \int_{0}^{\infty} A(\omega, \xi) \sqrt{\xi^{2}+\frac{j \omega}{\alpha_{s}}} \cos (\xi x) d \xi=\kappa_{p} \frac{\partial}{\partial y} T_{p}, \\
{[0<x \leqslant a ; y=0],}
\end{array}
$$

$\int_{0}^{\infty} A(\omega, \xi) \sqrt{\xi^{2}+\frac{j \omega}{\alpha_{s}}} \cos (\xi x) d \xi=0, \quad[a \leqslant x<\infty]$,

where $j=\sqrt{-1}$ and $\xi$ is the FC transformed variable $x$. The solution of the above is completed by using a discontinuous integral identity ${ }^{6}$ to recognize the required function $A(\omega, \xi)$. From here on, utilizing the remaining $\mathrm{BCs}$, an expression is obtained for the spatially averaged temperature field in the pyroelectric strip. A condition sine qua non for the measurement proposed is that the thermal diffusion length, $\mu_{s}$ $=\sqrt{\left(2 \alpha_{s}\right) / \omega}$, must be longer than the half-width of the strip (a); this imposes a requirement for low chopping frequencies. Under these conditions, the output voltage of the pyroelectric is approximately

$V(\omega) \approx \sqrt{\frac{\pi}{2} p Q_{a} H(\omega)} \int_{0}^{\infty} \frac{J_{1}\left(a \sqrt{\xi^{2}+\sigma_{s}^{2}}\right)}{\xi^{2}+\sigma_{s}^{2}} J_{0}(a \xi) d \xi$,

where $p$ is the pyroelectric coefficient and $K$ the dielectric constant of the sensor; $H(\omega)$ is the system frequency response; $\epsilon_{0}$ is the permittivity of free space; $J_{\nu}(z)$ is the Bessel function of the first kind of order $\nu ; \sigma_{i}=(1$ $+j) \sqrt{\omega /\left(2 \alpha_{i}\right)}=(1+j) / \mu_{i}$ with $i=p$ or $s$. Retaining only the first terms in the expansion of the Bessel functions and approximating the upper limit of the integral to $1 / a$, with $H(\omega)$ taken as equal to 1 , yields

$$
V(\omega) \approx-\frac{P}{\kappa_{s}}\left[\ln \omega+\ln \left(\frac{a^{2}}{\alpha_{s}}\right)+j \frac{\pi}{2}-\mathrm{const}\right] .
$$

The real part of this term is proportional to the logarithm of the modulation frequency (see also Ref. 7 ); $P$ comprises light

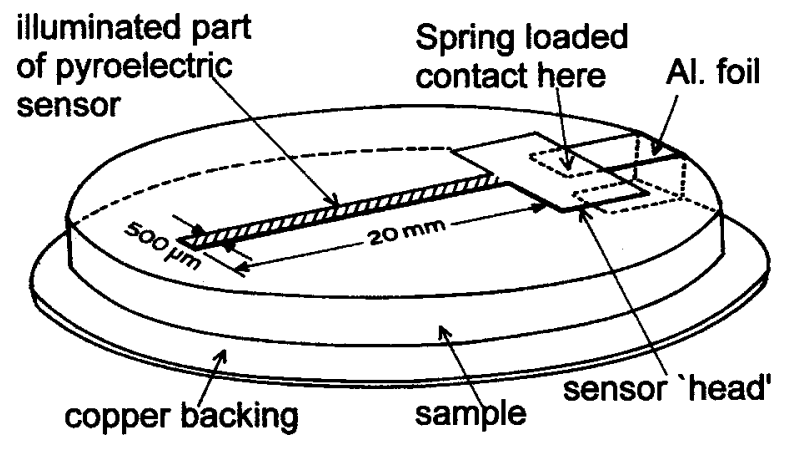

FIG. 2. Schematic of the pyroelectric strip and sample arrangement. A spring loaded contact (not shown) secures the assembly and forms the first signal electrode from the top metallization of the pyroelectric. The bottom metallization is extended electrically with aluminum foil $(<10 \mu \mathrm{m}$ thick $)$ to the copper backing which forms the second signal electrode. The entire assembly is placed in a vacuum chamber which is evacuated to better than $10^{-3}$ mbar. The hatched portion is illuminated with light focused using a cylindrical lens.

source and detector constants. The value of $\kappa_{s}$ of an unknown sample can thus be deduced from this linear region by normalizing out $P$ by making a run using a reference sample with well known $\kappa_{s}$.

The experiments were conducted in the arrangement shown in Fig. 2. The sensor/heater was cut into the design shown from a sheet of $9 \mu \mathrm{m}$ thick polyvinylidene fluoride (PVDF) foil, with thin metallizations on both faces. One face of the narrow strip was darkened with a layer of black ink, the thickness of which is estimated to be less than $1 \mu \mathrm{m}$; the "head" of the sensor which is not illuminated is left unpainted and is mainly to avail a sufficient area to obtain the electrical contacts to the external circuitry. The strip was mounted on the sample with a very fine veneer of

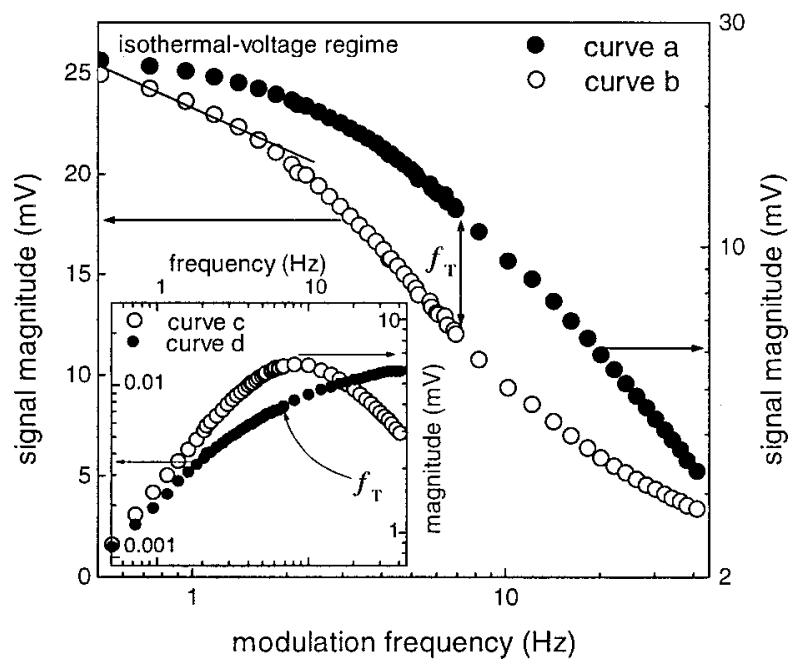

FIG. 3. Frequency responses of the pyroelectric strip (capacitance $C_{p}$ $\approx 1.3 \mathrm{nF}$ and resistance $R_{p} \approx 54.7 \mathrm{G} \Omega$ ) on a copper sample with variations in the electrical time constant $\tau_{E}$. The horizontal axes in the inset represent modulation frequency $(\mathrm{Hz})$; the vertical axes represent the signal magnitude $(\mathrm{mV})$. The input resistances $R_{\text {in }}$ used were $\approx 10^{12} \Omega$ [curve (a)], $10 \mathrm{M} \Omega$ [curve (c) in the inset] and $10 \mathrm{k} \Omega$ [curve (d) in the inset]. The corner frequency $f_{T}=\omega_{T} / 2 \pi$, due to the thermal time constant, is identified from curve (d) at the $-3 \mathrm{~dB}$ point and is also marked in curves (a) and (b). In curve (b), the voltage is plotted on a linear scale, with $R_{\text {in }} \approx 10^{12} \Omega$, and shows the "isothermal-voltage", regime in which the slope is used to calculate the thermal conductivity. 
Apiezon-M grease in between to ensure good thermal contact. Light from a $35 \mathrm{~mW} \mathrm{He}-\mathrm{Ne}$ laser operating at $632.8 \mathrm{~nm}$ was passed through a cylindrical lens to produce a line focus and uniformly illuminate the strip. Usual lock-in detection was employed to obtain the output voltage of the pyroelectric sensor as a function of the modulation frequency, which was swept by varying the speed of a mechanical chopper.

The system frequency response, $H(\omega)$, which characterizes the assembly, is dictated by corner frequencies $\omega_{T}$ and $\omega_{E}$. These are due, respectively, to the thermal time constant $^{8}\left(\tau_{T}=\omega_{T}^{-1}\right)$ which considers the sensor's "thermal environment,' and the usual electrical time constant ${ }^{8}\left(\tau_{E}\right.$ $=\omega_{E}^{-1}$ ) which considers the effective impedance of the sensor and the front end of its associated electronics. The thermal time constant is given by $\tau_{T}=C / G$, where $C$ is the heat capacity of the sensor and its associated components, and $G$ the thermal conductance coupling the sensor to a heat sink. In order to be able to clearly identify the linear output variation described by Eq. (6), a flat frequency response is expected of $H(\omega)$ in the low chopping frequency region. This could be ensured by using the sensor in the current mode ${ }^{2}$ of operation, when $\omega \tau_{E} \ll 1$ and concomitantly $\omega \tau_{T} \gg 1$. Modifications to $\tau_{E}$ can be made easily; manipulation of $\tau_{T}$ is inconvenient, but the case where $\omega \tau_{T} \gg 1$ is usually satisfied in conventional PPE experiments ${ }^{9}$ when the chopping frequency range is chosen such that $\omega_{T}$ lies below the lowest frequency. On the other hand, in the present STRIPPE technique, the requirement for low chopping frequencies causes $\omega_{T}$ to have a significant bearing on $H(\omega)$ well within the modulation frequency range. However, by utilizing the symmetry of the voltage responsivity ${ }^{8}$ of pyroelectric sensors with regard to the corner frequencies, the use of a high input impedance $\left(>10^{10} \Omega\right)$ preamplifier before the lock-in amplifier causes $\omega_{E}$ now to be pushed towards the lowest available frequency. By this it is possible to avail a range of frequencies $\tau_{E}^{-1}<\omega<\tau_{T}^{-1}$, where the output voltage profile described by Eq. (6) is preserved. This is the "isothermalvoltage", $(I-V)^{10}$ mode of operation, reported earlier in connection with pyroelectric based infrared detectors.

With this, interpretable frequency responses could be obtained with experiments conducted on representative samples. Copper was used as the reference sample and Fig. 3 shows the frequency responses obtained for different values of the electrical time constant $\left(\tau_{E}\right)$ obtained by varying the
TABLE I. Thermal conductivities of representative samples obtained using the STRIPPE technique with literature values provided for comparison.

\begin{tabular}{lccc}
\hline \hline & STRIPPE technique & & Literature values ${ }^{\mathrm{a}}$ \\
\cline { 2 - 2 } Sample & $\kappa_{s}$ & & $\kappa_{s}$ \\
& $\left(\mathrm{~W} \mathrm{~cm}^{-1} \mathrm{~K}^{-1}\right)$ & & $\left(\mathrm{W} \mathrm{cm}^{-1} \mathrm{~K}^{-1}\right)$ \\
\hline Copper & Reference & \\
& sample & 4 \\
Aluminum & 2.5 & 2.39 \\
Brass & 1.1 & 1.046 \\
Germanium & 0.55 & 0.602 \\
Silicon & 1.15 & 1.27 \\
\hline \hline
\end{tabular}

${ }^{\mathrm{a}}$ Reference 11 .

input impedance $\left(R_{\text {in }}\right)$ of the preamplifier. The slope of curve (b) in the region indicated, for various samples, was used to calculate their thermal conductivities. Good agreement was noted between values measured using the STRIPPE method and values quoted in the literature ${ }^{11}$ as shown in Table I.

Heartfelt thanks are due to Professor R. M. Vasu and Dr. G. Mohan Rao for the use of their respective laboratory facilities for the experiments. One of the authors (S.M.) is grateful to K. Deenamma Vargheese and G. Ganesh Chandan for their valuable support at every step of this work and to Joan Mollevanger and Dr. H. Manohar for the $9 \mu \mathrm{m}$ active element. He dedicates this work to his parents. The financial support of the Instrument Development Program, Department of Science and Technology, India is gratefully acknowledged.

${ }^{1}$ A. Mandelis and M. M. Zver, J. Appl. Phys. 57, 4421 (1985).

${ }^{2}$ M. Chirtoc and G. Mihăilescu, Phys. Rev. B 40, 9606 (1989).

${ }^{3}$ U. Zammit, M. Marinelli, R. Pizzoferrato, F. Scudieri, and S. Martellucci, Phys. Rev. A 41, 1153 (1990).

${ }^{4}$ D. Dădâralat, M. Chirtoc, C. Nemaţu, R. M. Candêa, and D. Bićanić, Phys. Status Solidi A 121, K231 (1990).

${ }^{5}$ I. N. Sneddon, Fourier Transforms, 1st ed. (McGraw-Hill, New York, 1951).

${ }^{6}$ Tables of Integral Transforms, edited by A. Erdélyi (McGraw-Hill, New York, 1954) Vol. I, p. 55, Eq. (37).

${ }^{7}$ D. W. Oxtoby, J. Chem. Phys. 85, 1549 (1986).

${ }^{8}$ E. H. Putley, in Semiconductors and Semimetals, edited by R. K. Willardson and A. C. Beer (Academic, New York, 1970), Vol. 5, Chap. 6.

${ }^{9}$ H. Coufal and A. Mandelis, Ferroelectrics 118, 379 (1991).

${ }^{10}$ M. Chirtoc, R. M. Candêa, and V. Mercea, Ferroelectrics 56, 283 (1984).

${ }^{11}$ Y. S. Touloukian, Thermophysical Properties of Matter (Plenum, New York, 1970). Vols. 1 and 2. 